\title{
3D Needle Shape Estimation in TRUS-Guided Prostate Brachytherapy Using 2D Ultrasound Images
}

\author{
Michael Waine ${ }^{1}$, Carlos Rossa ${ }^{1}$, Ron Sloboda ${ }^{2}$, Nawaid Usmani ${ }^{2}$, and Mahdi Tavakoli ${ }^{1}$
}

\begin{abstract}
In this paper we propose an automated method to reconstruct the 3D needle shape during needle insertion procedures using only 2D transverse ultrasound (US) images. Using a set of transverse US images, image processing and random sample consensus (RANSAC) is used to locate the needle within each image and estimate the needle shape. The method is validated with an in-vitro needle insertion setup and a transparent tissue phantom, where two orthogonal cameras are used to capture the true 3D needle shape for verification. Results showed that the use of at least 3 images obtained at $75 \%$ of the maximum insertion depth or greater allows for maximum needle shape estimation errors of less than $2 \mathrm{~mm}$. In addition, the needle shape can be calculated consistently as long as the needle can be identified in $30 \%$ of the transverse US images obtained. Application to permanent prostate brachytherapy (PPB) is also presented, where the estimated needle shape is compared to manual segmentation and sagittal US images. Our method is intended to help assess needle placement during manual or robotassisted needle insertion procedures after the needle has been inserted.
\end{abstract}

Index Terms-prostate, brachytherapy, ultrasound, needle, estimation

\section{INTRODUCTION}

$\mathbf{U}$ LTRASOUND (US) imaging is widely used to guide percutaneous needle insertion procedures because of its accessibility, low-cost, non-ionizing nature, and real-time capability. These benefits come at the cost of image quality; US images provide low soft-tissue contrast resolution compared to modalities such as magnetic resonance imaging or computed tomography. In addition, US images often contain speckle artifacts, shadows, reverberations, and mirror image artifacts that require proper interpretation to fully understand the image. It is challenging for surgeons to perform complex tasks while accounting for these imaging limitations, or for US-guided robotic systems to distinguish US artifacts from intended targets. For these reasons, needle segmentation and shape estimation from US images is a challenging and important research topic.

This work was supported by the Natural Sciences and Engineering Research Council (NSERC) of Canada under grant CHRP 446520, the Canadian Institutes of Health Research (CIHR) under grant CPG 127768, and by the Alberta Innovates - Health Solutions (AIHS) under grant CRIO 201201232.

1 Michael Waine, Carlos Rossa and Mahdi Tavakoli (Corresponding Author) are with the Department of Electrical and Computer Engineering, University of Alberta, AB, Canada T6G 2V4. E-mail: mwaine@ualberta.ca; rossa@ualberta.ca; mahdi.tavakoli@ualberta.ca

2 Ron Sloboda and Nawaid Usmani are with the Cross Cancer Institute and the Department of Oncology, University of Alberta, Edmonton, AB, Canada T6G 1Z2. E-mail: \{ron.sloboda, nawaid.usmani\}@albertahealthservices.ca.
Various research studies have been performed on automatic object segmentation from 3D volumetric US images. Several methods have implemented the Radon and Hough transforms. For segmentation of surgical instruments, Novotny et al. [1] used a graphics processing unit operated in parallel architecture to perform their Radon transform-based method in real-time. Zhou et al. [2] and Qiu et al. [3] used a coursefine search strategy to allow for fast implementation of the 3D Hough transform for segmentation of straight needles [4]. Qiu et al. [5] also used phase-grouping combined with either least-squares or 3D Hough transform optimization for realtime needle shape segmentation. Research on curved needle segmentation from 3D US includes a generalized Radon transform with Bezier curves [6], and the Hough transform combined with ray casting and polynomial approximation [7]. Alternatives to Radon/Hough transform-based methods include difference imaging techniques [8], [9] or RANSAC (random sample consensus)-based methods [10]-[12].

The above approaches make use of 3D volumetric US images. Although some 3D US probes make use of 2D arrays to provide real-time 3D US, the majority of current clinical 3D US machines rely on sweeping a 1D array to produce a $3 \mathrm{D}$ volume. This technology is limited by slower frame rates and bulkier transducers compared to 2D US machines [13]. In addition, real-time 3D transrectal US (TRUS) probes are costly and are currently not widely available for use in clincal prostate brachytherapy. For this type of procedure, 2D US imaging methods are simpler to implement and allow for fast image processing capabilities.

Most 2D US needle segmentation methods require images where the needle is visualized within the plane of the US beams, as shown in Fig. 1a. These are referred to as sagittal images [14]. Examples that use 2D sagittal US images include work performed by Kaya et al. [15], [16], who combined Gabor filtering and RANSAC to estimate linear needle shapes, and work by Okazawa et al. [17], who used the Hough transform to estimate curved needle shapes. In addition, Ayvaci et al. [18] performed biopsy needle segmentation on TRUS videos for use in MRI/TRUS fusion guided biopsy. Work on needle tip tracking has been performed by Mathiassen et al. [19], who developed an optical tracking system based on intensity features in the images and Neubach et al. [20], who used a $30^{\circ}$ needle-probe setup to improve visualization of the needle tip, and utilized image subtraction of subsequent frames for their needle steering robot.

Although sagittal US images can facilitate rapid visual- 


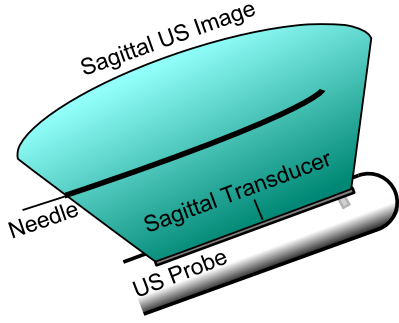

(a) Sagittal US Imaging

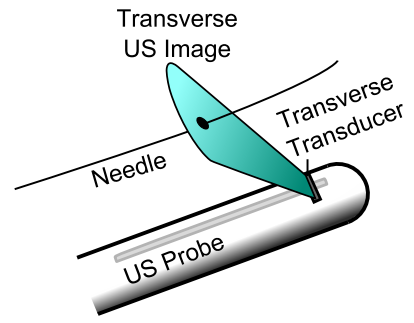

(b) Transverse US Imaging
Fig. 1. Example showing the difference between a) sagittal US imaging and b) transverse US imaging. Many clinical TRUS probes contain two transducer arrays to allow for both sagittal and transverse US imaging.

ization of the needle, in practice it is difficult to align the US transducer with the plane in which the needle deflects [21]. Additionally, alignment may not always be possible; for example, TRUS probes are limited to two degrees of freedom: translation parallel to the TRUS probe, and rotation about its sagittal axis. This limitation may prevent some needle shapes from being properly visualized, considering that the needle can deflect in any sagittal image plane, or even multiple planes when rotation is introduced.

In contrast to sagittal US imaging, images obtained perpendicular to the US transducer array, as in Fig. 1b, are known as transverse or axial US images; we will use the former term throughout the rest of this paper. Transverse imaging is widely used in prostate brachytherapy for several reasons. It provides surgeons with a consistent view of the prostate, regardless of where the needle was inserted, unlike sagittal imaging where the probe must be rotated after each insertion in order to locate the needle. As a result, transverse images are often used for pre-implant treatment planning and image registration during prostate brachytherapy [22]. Prostate brachytherapy equipment and software, such as the US stepper and the physical and electronic template grids are also designed for transverse imaging, making these type of images attractive for clincal use. However, in transverse images, only a cross-section of the needle is visible. Recent techniques have been developed by Greer et al. [23] and Adebar et al. [24], which integrate transverse US imaging with Doppler US for robotic needle steering. A voice coil actuator was attached to the needle to induce small vibrations that allow the needle to be seen in Doppler US. As well, Vrooijink et al. [25], [26] developed a method for real-time needle tip tracking using a motorized US probe. Another technique developed by Yan et al. [27] made use of difference imaging and shape-based level set segmentation for needle shape identification using transverse US images.

In this paper, we demonstrate a real-time needle segmentation algorithm based on 2D transverse images. Our work is an in-depth exploration of our initial feasibility assessment performed in [28]. We implement modifications to handle effects of needle rotation and use a-priori knowledge of the needle's initial position and orientation to reduce the number of images required for the technique. As well, we test the algorithm on both in-vitro phantom tissue experiments and in-vivo prostate brachytherapy images. Unlike [23]-[26], our method does not require the use of specialized needle attachments and can be readily incorporated with current clinical equipment. In contrast to [27], we used in-vivo prostate brachytherapy images and a threshold-based RANSAC approach to cope with the large amounts of noise and background objects present within clinical images. We also explored the number and depth at which the transverse images should be obtained for accurate needle shape estimations. Using a series of transverse US images, we apply image processing and a RANSAC algorithm to estimate the entire needle shape from the base of the needle to its tip. RANSAC has been successfully used for needle segmentation applications in 3D US images [10]-[12] and sagittal US images [15], but so far has not been applied to multiple transverse US images using in-vivo images.

By incorporating known spatial constraints on the needle along with a 3rd order polynomial approximation of the needle shape, we can obtain reliable estimations of the entire needle shape for clinical applications. Third-order polynomials have been shown to provide a good approximation of surgical needle shapes [11], [17], [23], [24], [29] and prevent unrealistic rippling effects caused by higher-order polynomials. In addition, a low-order polynomial model is less computationally intensive for the RANSAC procedure compared to more complex, physics-based models. At the same time, our approach can cope with issues that complicate both manual and automated transverse US needle segmentation, such as cases where the acoustic shadows camouflage the needle [12], or cases where the needle is difficult to distinguish from other nearby hyperechoic objects.

Insertion experiments are carried out on phantom tissue to validate our proposed method and to determine the depth at which the images should be obtained as well as the overall image quality necessary to obtain accurate estimates. Analysis of clinical US images collected from human prostate brachytherapy procedures using the proposed method is also performed.

The rest of the paper is organized as follows. Section II describes the process used to identify the needle within each transverse US image. In Section III, the RANSAC algorithm used to estimate the 3D needle shape is described. Section IV details the experimental setup and the phantom tissue experiments used to validate the accuracy of the proposed method. Experimental results are discussed in Section V. In Section VI, applications with clinical data are demonstrated and discussed.

\section{Locating NeEdle Point CAndidates}

During permanent prostate brachytherapy (PPB), transverse US images of the needle embedded in tissue resemble Fig. 2. The needle appears as a hyperechoic object often followed by a comet-tail artifact [14]. Other hyperechoic objects may be visible in addition to the needle. For example, in Fig. 2, gel inserted into the urethra causes it to appear brighter than the needle itself, which allows the urethra to be used for landmarking purposes. Since planning is often performed with the urethra aligned in the centre of the ultrasound images, it is a useful landmark to identify during prostate brachytherapy and helps with identification of the prostate. In addition, 


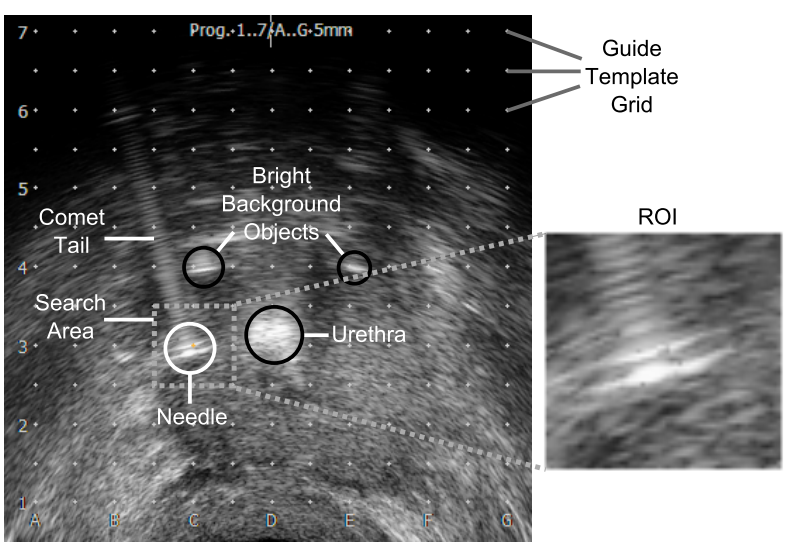

Fig. 2. An example of the needle appearance in a $2 D$ transverse US image obtained during PPB. The needle is circled in white and extraneous objects are circled in black. The ROI is shown on the right.

brachytherapy seeds and imaging noise can produce bright US intensities similar to the intensities caused by the needle. A study done by Wen et al. [30] showed that brachytherapy seeds could be segmented using similar techniques used in needle segmentation. Therefore, image processing must be performed to differentiate the needle from other hyperechoic objects or noise. Doing so involves defining a region of interest (ROI) and applying image enhancement techniques. It is assumed that all of the transverse US images used in the analysis were obtained along the needle, and correspond to either the needle shaft or the needle tip. This is confirmed by looking for a special double reflection feature that corresponds to the needle tip known as a "hamburger" signature before the transvere images are obtained from the US machine. As well, it is assumed that the depth of the needle within the tissue is also known. This is confirmed by recording the depth of the needle tip observed from the US machine before the transverse images are obtained.

\section{A. Definition of the Region of Interest (ROI)}

An example of a manual PPB setup is shown in Fig. 3. A template grid is used in the procedure to guide the needle towards the intended target [22]. An example showing the template grid superimposed on a transverse US image is shown in Fig. 2.

A dynamic region of interest (ROI) algorithm is used, based on methods proposed in [20], [27]. First, a large ROI is selected in order to initially locate the needle, based on the template grid insertion location and the expected needle deflection at the initial depth of the transverse US images. This initial ROI for the in-vitro experiments was chosen as a $30 \mathrm{~mm}$ by $10 \mathrm{~mm}$ window and the ROI for the in-vivo patient study described in Section VI was chosen as a $10 \mathrm{~mm}$ by $10 \mathrm{~mm}$ window. This variation was due to the a-priori knowledge of typical needle deflections observed for each case. The initial ROI for one of the clinical US images is shown and magnified in Fig. 2. This ROI is applied to the first three consecutive US images nearest to the needle base, and needle point candidates are identified within the images using the method described in Section II-C. The needle location is then estimated using

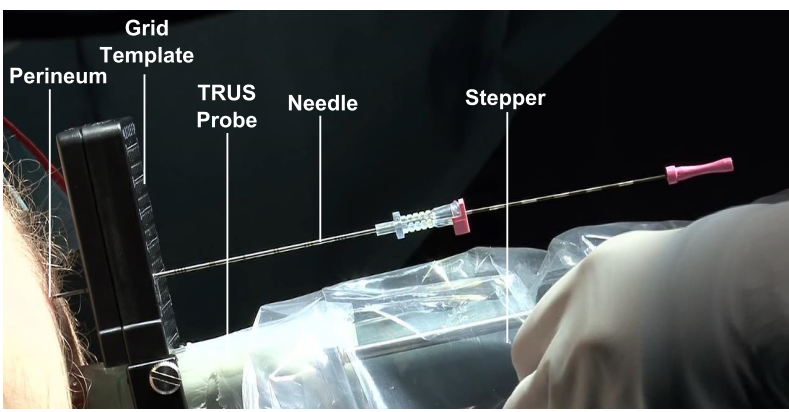

Fig. 3. An example of a typical PPB setup. A stepper operated by the surgeon controls the depth of the US probe. A template grid is used to guide the needle towards the intended target during the procedure.

the method described in Section III. If less than $30 \%$ of the expected needle point candidates are identified in the set of three images, the initial ROI is used again in the next set of three US images. Otherwise, the ROI is updated every three US images, which provides a fair trade-off between estimation accuracy and processing time. The updated ROI's are smaller than the originals (a $10 \mathrm{~mm} \times 10 \mathrm{~mm}$ window for the in-vitro images and a $5 \mathrm{~mm} \times 5 \mathrm{~mm}$ window for the in-vivo images), which further reduces the amount of extraneous background objects located within the ROI. The updated ROI is centered around the needle location in the most recent image.

\section{B. Contrast Enhancement}

An intensity transformation $T(r)$ is applied to the ROI to improve contrast between the needle and the background. The transformation is

$$
T(r)=r_{\min }+\left(r_{\max }-r_{\text {min }}\right)\left(\frac{r-r_{\text {low }}}{r_{\text {high }}-r_{\text {low }}}\right)^{\gamma}
$$

where $r, r_{\min }, r_{\max }, r_{\text {low }}, r_{\text {high }}$ represent normalized intensity values in the range $[0,1]$. Here 0 represents the color black and 1 represents the color white. The values of $r$ correspond to the pixel intensities contained in the original image. The range $\left[r_{\min }, r_{\max }\right]$ defines the minimum and maximum intensities desired in the transformed image. The values $r_{\text {low }}$ and $r_{\text {high }}$ define saturation thresholds. Through equation (1), pixels in the ROI having intensities less than $r_{\text {low }}$ are given the intensity $r_{m i n}$ in the transformed ROI. Likewise, pixels with intensities larger than $r_{h i g h}$ are given the intensity $r_{\max }$.

For our purposes, the desired spectrum is chosen to span $[0,1]$ and the values $r_{l o w}$ and $r_{h i g h}$ are set to $10 \%$ and $100 \%$ of the maximum intensity values present within the original ROI, respectively, causing a single-sided saturation of the (dark) background pixels. The value of $\gamma$ specifies the shape of the exponential curve that maps the intensity values from the original ROI to the transformed ROI [31]. We set $\gamma>1$ in order to apply additional weight towards lower intensity pixels, allowing for increased contrast between the needle and background. This combination of ROI and contrast enhancement yields consistent results, especially for the 


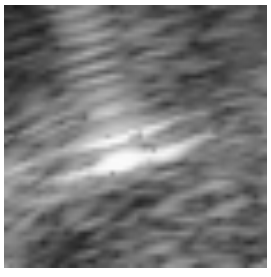

(a) Original Image

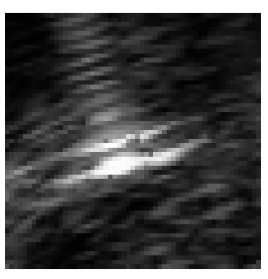

(b) Intensity Transform

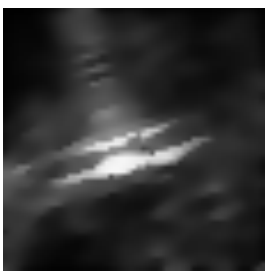

(c) Anisotropic Diffusion
Fig. 4. Comparison between a) the original image and b) the result after applying the intensity transform and the anisotropic diffusion filter. Background noise is suppressed while the high contrast between the needle and background is retained.

standard distances used in prostate brachytherapy (typically less than $50 \mathrm{~mm}$ between the needle and the US probe).

To reduce computation time, we end the image processing stage at this point. For applications where computational complexity is not an issue, we have found that the anisotropic diffusion filter developed by Perona et al. [32] reduces the majority of the background noise present in the ROI. The anisotropic diffusion technique is an adaptive filter that prioritizes intra-region smoothing over inter-region smoothing, thereby reducing noise while preserving edge details. This allows for the suppression of noise within the ROI while retaining the stark contrast between the hyperechoic needle cross-section and the background. Figure 4 shows a comparison between the original ROI, the result after applying contrast enhancement, and the result after applying both contrast enhancement and the anisotropic diffusion filter. More recent filtering techniques used in the literature such as speckle reducing anistropic diffusion [33] or detail preserving anisotropic diffusion [34] were considered, but the advantages they provide are not applicable for our purposes of needle enhancement in transverse US images.

\section{Candidate Needle Point Identification}

After all image processing steps have been performed, a cumulative histogram of the pixel intensities in the ROI is obtained. The histogram is used to determine an intensity threshold $\alpha$ that corresponds to the $n$ brightest pixels in the ROI, where $n$ is defined by:

$$
n=\frac{\beta A}{r_{d x} r_{d y}}
$$

where $A$ is the area of the needle cross-section in $\mathrm{mm}^{2}, r_{d x}$ and $r_{d y}$ are the height and width in mm of a single pixel, and $\beta$ is a tunable parameter to account for deterioration of the needle shape in the US image due to scattering and diffraction. In our work, we selected $\beta$ to be 0.75 based on emperical results, meaning that $75 \%$ of the needle is expected to be visible in the US image. Pixels with intensities larger than $\alpha$ are considered as candidate needle points within the transverse US images and these candidate needle points are identified for each transverse image obtained along the needle. A flowchart showing the image processing steps is shown in Fig. 5.

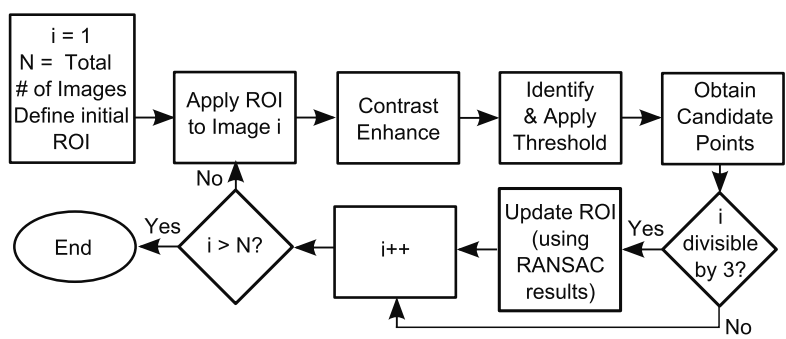

Fig. 5. Flowchart summary of image processing procedure. For each transverse image, the ROI is applied, and image processing is used to locate needle point candidates. Every three images, RANSAC is applied to the needle point candidates and the results are used to update the ROI. The process continues until all images have been analyzed.

Note that equation (2) provides a liberal estimate of the candidate needle points, since needle visibility is not yet taken into account. At this stage, it is difficult to determine which, if any, of the candidate needle points should be considered as outliers. A robust RANSAC-based method for detecting outliers based on the relative positions of the candidate needle points within each image along with the corresponding needle visibility in each image is described in the following section.

\section{NeEdle Shape Estimation Using RANSAC}

There has been a variety of research performed in the area of needle steering during percutaneous needle insertion procedures [35]-[37]. Needle steering techniques exploit the fact that a needle with a bevelled tip follows a curved trajectory during insertion. Rotating the needle causes the position of the bevelled tip to change, which changes the needle's trajectory. Based on observation of the steering techniques employed in our patient study, it is assumed that the needle rotates at most once during the insertion process. Therefore, we use a thirdorder polynomial to estimate the needle beginning from the template grid and ending at the needle tip.

The needle shape estimation algorithm used in this paper is composed of two steps: 1) fit the needle point candidates using RANSAC, and 2) optimize the solution to the previous step via a weighted least squares regression.

\section{A. Fitting the Needle Point Candidates using RANSAC}

RANSAC is a robust model-fitting algorithm developed by Fischler and Bolles to smoothen data containing a significant proportion of gross errors [38]. For example, in our case, calcifications, brachytherapy seeds, and microbubbles can produce false needle point candidates within the ROI defined in Section II. If these objects are not intelligently removed, they can greatly influence the curve fit generated by a method such as Ordinary Least Squares Regression. The RANSAC Toolbox developed in [39] is used for implementation of the proposed algorithm.

We apply RANSAC multiple times so that the ROI described in II-A can be constantly updated. For every three US images, a new RANSAC procedure will be applied, and the needle point candidates identified from previous applications are carried forward. 
The fundamental concept behind RANSAC involves iteratively sampling the minimum number of data points required for model estimation and comparing this estimation to the entire set of data points. A good model estimation corresponds to the model which fits the largest proportion of the data. Assume we have a model $M$ with $k$ solvable, free parameters. As well, assume we have a set $S$ containing $N>k$ data points. A random selection of $k$ points from $S$ are sampled and used to solve for $M$. In our case, $S$ refers to the combined set of needle point candidates identified in the transverse images analyzed so far, and $M$ is the needle shape model. The needle shape model used for our work is defined by the following equations:

$$
\begin{gathered}
x(d)=a_{3} d^{3}+a_{2} d^{2}+a_{1} d+a_{0} \\
y(d)=b_{3} d^{3}+b_{2} d^{2}+b_{1} d+b_{0} \\
z(d)=d
\end{gathered}
$$

The value $d$ represents the depth with respect to the grid template. The values $\left(a_{3}, a_{2}, a_{1}, a_{0}\right)$ and $\left(b_{3}, b_{2}, b_{1}, b_{0}\right)$ are the free parameters of the needle shape model. The $(x, y, z)$ axes are defined in Figure 6 with the coordinate frame origin at the intersection of the needle and the face of the grid template.

Because the needle is being inserted through the grid template, equations (2)-(4) can be simplified by incorporating the insertion location information into the model. In this work, the grid template is positioned to be exactly perpendicular to the ultrasound transducer and the calculated needle shape axes are aligned with the grid template. If the template is not perpendicular to the ultrasound transducer, a coordinate transformation matrix would be required to transform the image frames to the grid template frame before continuing with the procedure. This can be easily calculated if the position of the template with respect to the transducer is known. We assume the portion of the needle passing through the grid template holes is constrained to be approximately parallel to the $z$-axis. These constraints and the simplified model are summarized below:

$$
\begin{gathered}
x(0)=x_{0}, y(0)=y_{0}, \dot{x}(0)=0, \dot{y}(0)=0 \\
x(d)=a_{3} d^{3}+a_{2} d^{2}+x_{0} \\
y(d)=b_{3} d^{3}+b_{2} d^{2}+y_{0}
\end{gathered}
$$

where $\dot{x}(d)$ and $\dot{y}(d)$ represent the first derivatives of equations (2)-(3). Equation (4) remains unchanged. In our case, equations (6)-(7) require $k=2$ samples from $S$ to solve for the free parameters. Let us refer to this parameterized model as $M_{1}$.

The values $\left(x_{i, j}, y_{i, j}, d_{i, j}\right)$ identify the $3 \mathrm{D}$ coordinates of the $i^{t h}$ needle point candidate found within the $j^{t h}$ transverse image. The pair $\left(x_{i, j}, y_{i, j}\right)$ refers to point $i$ 's spatial coordinates within the $j^{t h}$ transverse image and $d_{i, j}$ refers to the depth of point $i$ with respect to the grid template.

After solving for $M_{1}$, we identify the subset of points in $S$ that adequately satisfies $M_{1}$. This subset is known as the consensus set $C S_{1}$ corresponding to $M_{1}$. CS $S_{1}$ is determined from

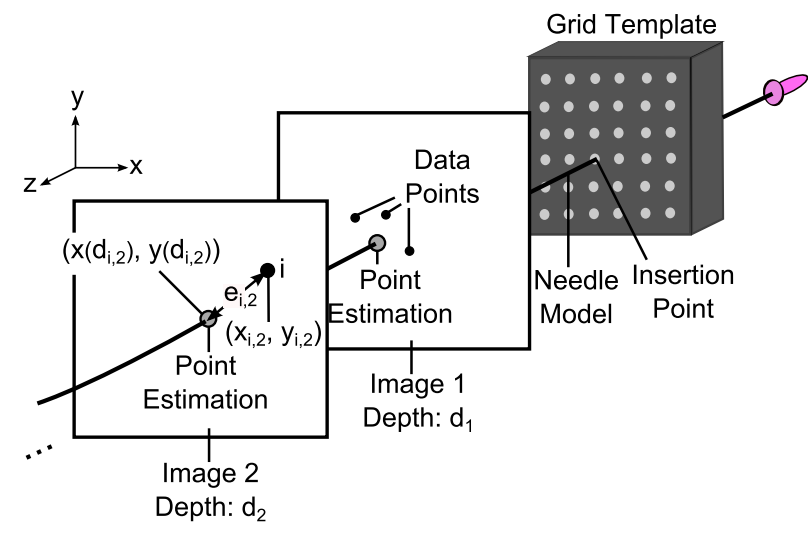

Fig. 6. Diagram showing $e_{i, 2}$ for the $i^{t h}$ point in the data set $S$.

$$
C S_{1}=\left\{\left(x_{i, j}, y_{i, j}, d_{i, j}\right) \mid e_{i, j}\left(d_{i, j}\right)<\tau\right\}
$$

where

$$
e_{i, j}\left(d_{i, j}\right)=\sqrt{\left(x_{p_{2}}\left(d_{i, j}\right)-x_{i, j}\right)^{2}+\left(y_{p_{2}}\left(d_{i, j}\right)-y_{i, j}\right)^{2}}
$$

The value $\tau$ specifies the inlier tolerance. We selected $\tau$ to be $0.635 \mathrm{~mm}$, which is equal to the radius of an 18-gauge brachytherapy needle. The residual error $e_{i, j}$ between the point $i$ and the model $M_{1}$ is used to identify the needle points that satisfy the model.

Next, a new iteration is performed, where the model is re-parameterized using a new random sample of $k$ points to obtain $M_{2}$ and calculate the corresponding consensus set $C S_{2}$. This procedure is performed a minimum of $N_{c}$ times, and a cost function is evaluated for each model and consensus set pair. The M-Estimator Sample Consensus (MSAC) technique developed by Torr et al. [40] is used to identify the model that minimizes the cost function

$$
C=\sum_{j=1}^{N_{I}} \sum_{i=1}^{n_{j}} f\left(e_{i}\right), f\left(e_{i}\right)= \begin{cases}e_{i}, & e_{i}<\epsilon \\ \epsilon, & e_{i} \geq \epsilon\end{cases}
$$

where $N_{I}$ is the total number of transverse images analyzed so far, $n_{j}$ is the number of needle point candidates in the $j^{t h}$ image, and $\epsilon$ is the cost function termination threshold. The consensus sets are ranked according to the inverse of their cost function magnitude. Once the change in the cost function for the highest ranked consensus set becomes smaller than $\epsilon$, and at least $N_{c}$ consensus sets have been obtained, the process terminates.

At this point, a subset of the original needle point candidates has been identified; this subset excludes false positives. An initial curve fit describing the needle shape has also been identified. To summarize the RANSAC procedure thus far: two randomly-selected points from the data set are used to fit the needle shape model. Next, a consensus set is found, which includes needle point candidates that adequately satisfy the fitted model. Then, the quality of the model and corresponding consensus set is evaluated using MSAC. This is performed until a model with sufficient quality has been identified. 


\section{B. Optimize Results}

The needle shape identified using the RANSAC procedure necessarily passes through at least 2 points as a result of Section III-A. We optimize the curve fit by applying a weighted least squares regression to the consensus set identified using the RANSAC algorithm. This is performed by minimizing the error estimates

$$
\begin{aligned}
& s_{x}=\sum_{j=1}^{N_{I}} \sum_{i=1}^{n_{j, C S}} w_{i, j}\left(x_{p_{2}}\left(d_{i, j}\right)-x_{i, j}\right)^{2} \\
& s_{y}=\sum_{j=1}^{N_{I}} \sum_{i=1}^{n_{j, C S}} w_{i, j}\left(y_{p_{2}}\left(d_{i, j}\right)-y_{i, j}\right)^{2} \\
& w_{i, j}=\frac{(q-p)\left(r_{i, j}-r_{\min , C S}\right)}{r_{\max , C S}-r_{\min , C S}}+p
\end{aligned}
$$

where $n_{j, C S}$ is the number of data points in the $j^{t h}$ transverse image of the consensus set, $s_{x}$ and $s_{y}$ are the error estimates with respect to $x$ and $y$ respectively, and $w_{i, j}$ is the weighting applied to the $i^{\text {th }}$ data point of the $j^{t h}$ image. The value $r_{i, j}$ represents the intensity of the corresponding data point in the consensus set after the contrast enhancement steps described in Section II-B have been applied, $r_{m i n, C S}$ and $r_{\max , C S}$ represents the minimum and maximum intensities within the consensus set respectively, and $[p, q]$ defines the desired range of the weighting values. Equations (12)-(14) give greater weighting to higher intensity pixels, which are more likely to correspond to the needle. For simplicity, the maximum intensity was given a weight of 1.0 and the minimum intensity was given a weight of 0.5 . After the weighted least squares regression is applied to the data set, we obtain a 3D approximation of the needle shape starting from the template grid and ending at the most recent transverse image analyzed.

Note that the RANSAC procedure is applied multiple times in order to update the ROI between each application. In the first application of the RANSAC procedure, only the three transverse US images nearest to the needle base are analyzed, and inliers are obtained for each of these images. In the second application of RANSAC, the ROI is adjusted based on the most recent estimation of the needle location, and a new RANSAC procedure is applied to the next three transverse US images with the inliers from the previous application being carried forward and the outliers being removed. This process repeats until all of the transverse US images have been analyzed.

The result is a $3 \mathrm{D}$ curve representing the needle shape beginning from the grid template and ending at the needle tip. An example is shown in Fig. 7, where the inliers and outliers represent true and false needle point candidates respectively, and the optimized curve fit is the needle shape obtained from equations (11)-(13).

\section{EXPERIMENTAL SETUP}

The setup used to validate the proposed method is shown in Fig. 8a, and a sketch of setup mechanics is shown in Fig. 8b. An 18-gauge, bevel-tipped brachytherapy needle model PSS1820EZ (Worldwide Medical Technologies, Oxford, CT, USA) is manually inserted through a stabilizing

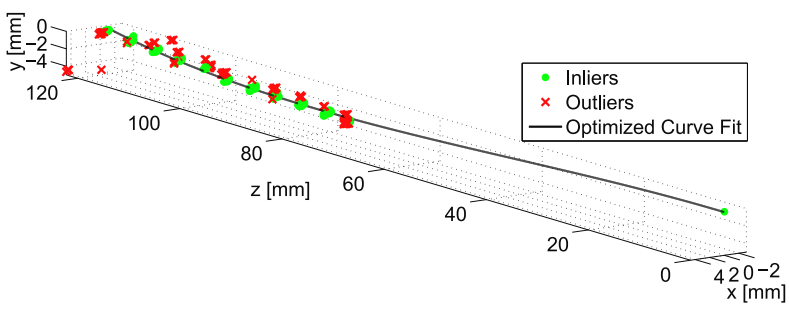

Fig. 7. 3D needle shape estimation using the proposed method. The inliers represent the consensus set found using the RANSAC algorithm and the optimized curve fit is found through weighted least squares regression.

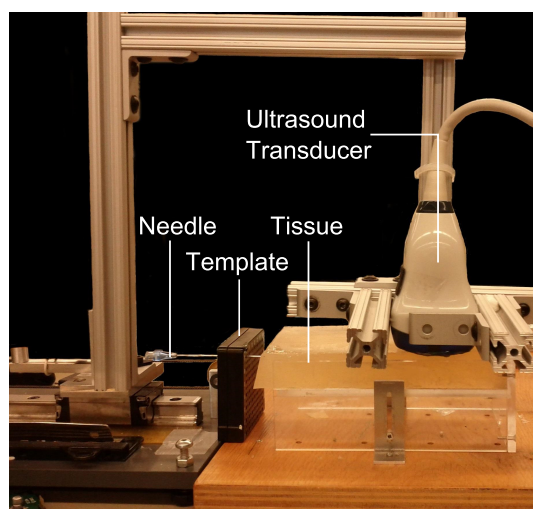

(a) Photograph of experimental setup

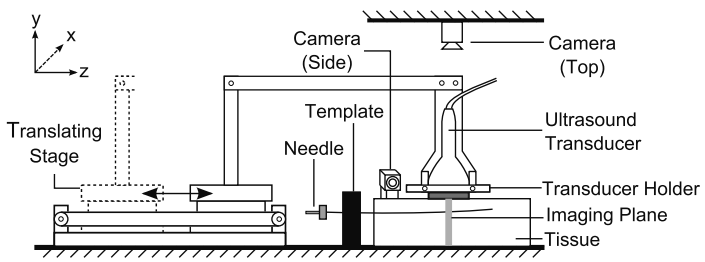

(b) Setup mechanics

Fig. 8. a) The experimental setup and b) depiction of the setup mechanics. The US transducer is attached to a translating platform, which is manually adjusted to obtain axial slices of the embedded needle every $5 \mathrm{~mm}$ of depth.

template grid to a depth of $125 \mathrm{~mm}$ in a transparent tissue phantom created using a plastisol formula of two parts plastisol to one part plastic softener (M-F Manufacturing Company, Fort Worth, TX, USA). During insertion, the needle is manually rotated $90^{\circ}$ at approximately half of the maximum insertion depth to simulate clinical practice. A layer of US gel is applied to the top of the tissue phantom to ensure the US transducer probe makes appropriate contact with the tissue. A single-degree-of-freedom (DOF) translating platform is used to move the US probe. A mechanical holder is used to secure a linear US transducer model 4DL14-5/38 (Analogic Ultrasound, Richmond, BC, Canada) to the translating stage. The stage is manually positioned to allow for the collection of transverse US images of the needle every $5 \mathrm{~mm}$ along the $\mathrm{z}$ axis, starting from a depth of $5 \mathrm{~mm}$, mirroring clinical practice.

A total of 25 transverse images are collected along the needle length using a SonixTouch Ultrasound System (Analogic Ultrasound, Richmond, BC, Canada). As well, two XCD-SX90 cameras (Sony, Park Ridge, NJ, USA) are used to capture the true 3D needle shape. One camera is mounted above the setup to obtain a view of the $(x, z)$ plane and the second camera is 
mounted at the side to obtain a view of the $(y, z)$ plane.

Needle segmentation from the camera images is performed using MATLAB. Points corresponding to the needle are manually identified in the images obtained from the two cameras and a third-order polynomial in each of the $(x, z)$ and $(y, z)$ planes is used to obtain the 3D curve fit. This manually segmented needle shape is used for verification of the RANSAC algorithm results obtained from Section III.

\section{Results \& Discussion}

Two separate tests using the in-vitro US images obtained with the setup described in Section IV are performed. The first test explored the optimal depths to obtain the transverse US images. The second test explored how the quality of the US images affected the needle shape estimation.

\section{A. Effects of Image Depth on Needle Shape Estimation}

The model described in Section III requires at least two different transverse images in order to characterize the needle shape. The 25 transverse images obtained from the experiment are grouped into subsets ranging from 3 successive slices to 25 successive slices, resulting in a total of 276 different image sets. For this stage of analysis, only successive slices were investigated in order to limit the number of image set combinations explored. The depth of the final slice is recorded for each image set, along with the "depth differential", which is found by subtracting the maximum transverse image depth from the minimum transverse image depth. As well, for each image set, the residual error between the estimated needle shape obtained using the proposed method and the measured needle shape obtained using the camera images is calculated. This error is calculated every $0.01 \mathrm{~mm}$ along the needle and the average error is reported. Associated contour plots are shown in Fig. 9, where the maximum resultant error plot is shown in panel a) and the average resultant error plot is shown in panel b).

The contour plots show the needle shape error associated with each image set, where each image set is identified by its final slice depth, and its depth differential. The best results are those in dark blue, while poor results are those in dark red. The optimal combinations of depth differential and final slice depth for the maximum error contour plots are outlined in Fig. 9a. Needle shape errors of $2 \mathrm{~mm}$ or less are obtained when the final slice occurs at a depth of at least $95 \mathrm{~mm}$, roughly $75 \%$ of the maximum insertion depth. Additionally, the depth differential has a significantly smaller effect on needle shape error than does the depth of the final slice. In general, as the depth of the final slice increases, the maximum needle shape error decreases, regardless of the depth differential. The exception occurs for very small depth differentials of less than $10 \mathrm{~mm}$, which corresponds to image sets containing less than 3 images. There is likely not enough information contained in such small image sets to accurately characterize the needle shape.

The average error contour plot from Fig. 9b demonstrates that the overall needle shape estimation can be determined with an average error of $1 \mathrm{~mm}$ or less when the final slice

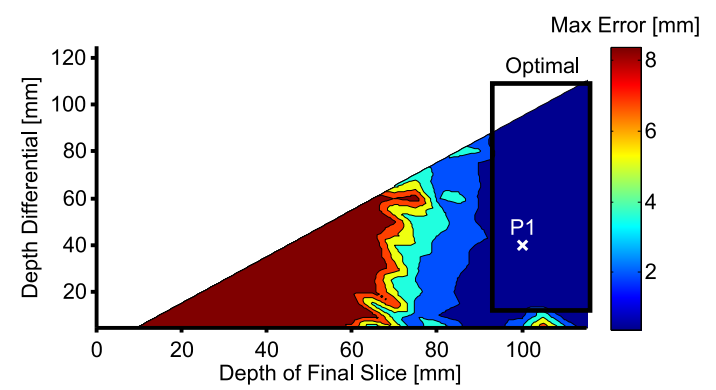

(a) Maximum resultant error

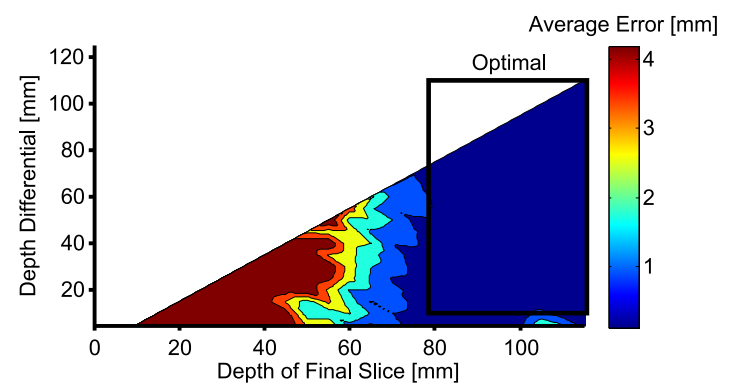

(b) Average resultant error

Fig. 9. Contour plots showing the a) maximum error and b) average error obtained for each of the 276 transverse image sets. Example point $\mathrm{P}$ demonstrates the specific transverse image set whose final slice ends at 100 $\mathrm{mm}$ and contains a depth differential of $60 \mathrm{~mm}$. Optimal combinations of depth differential and final slice depth are highlighted.

occurs at a depth of at least $80 \mathrm{~mm}$, regardless of depth differential. Again, the exception occurs for depth differentials of $10 \mathrm{~mm}$ or smaller. As well, the optimal results cover a larger area than those from Fig. 9a, demonstrating that the average needle shape error is quite robust with respect to the final slice depth.

The results overall demonstrate that the smallest errors are obtained with transverse image sets containing depth differentials of at least $10 \mathrm{~mm}$, corresponding to 3 transverse slices, and where the final slice depth is at least $75 \%$ of the maximum depth.

\section{B. Effects of Image Quality on Needle Shape Estimation}

In clinical transverse US images, there are a variety of complications that affect identification of the needle. For example, in our patient study, of the 615 images obtained, the needle could be precisely identified through manual segmentation in 412 images, $67 \%$ of the total number. Of the remaining $33 \%$, we found four major causes hindering needle identification: 1) the needle is not visible, 2) the needle image is distorted, 3) there are additional needle-like objects nearby, 4) a hyperechoic object is visible, but it does not have needlelike characteristics. An example of each case is shown in Fig. 10. Potential needle pixels identified using a simple thresholding algorithm are also highlighted in the figure, demonstrating how these issues adversely affect automated thresholding algorithms.

Transverse images where the needle cannot be clearly identified by traditional methods are denoted as "confounding" images. A method was developed to test the capabilities of 


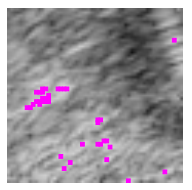

(a) Hidden

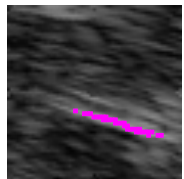

(b) Distorted

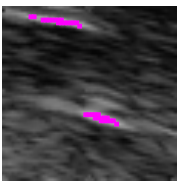

(c) Multiple

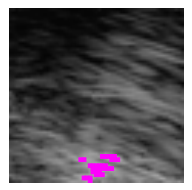

(d) Unclear
Fig. 10. Samples of ROI's demonstrating four major issues affecting needle identification. In a) the needle blends in with the surrounding tissue. In b) the needle can be seen, but its shape is distorted (elongated in this case). In c) there are two hyperechoic objects in the ROI. In d) a hyperechoic object is partially visible, but it is unclear whether it belongs to the needle.

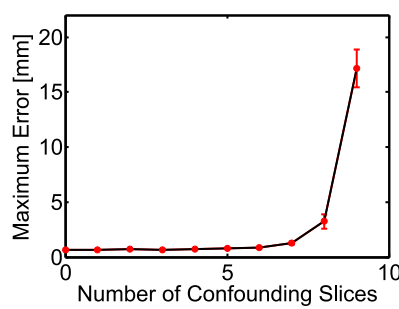

(a) Maximum Error

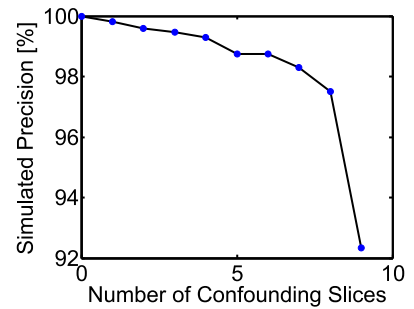

(b) Precision
Fig. 11. Effects of confounding slices on a) maximum error and b) precision The number of confounding slices was varied from 0 to 9 . In a), the mean and standard error obtained as a result of 100 simulations are plotted. In b) the precision over the course of 100 simulations is plotted.

our proposed algorithm to withstand the occurrence of these confounding images, specifically the type demonstrated in Fig. 10a where the needle cannot be identified at all. Based on the results of Section $\mathrm{V}-\mathrm{A}$ along with the fact that the prostate typically spans $45-60 \mathrm{~mm}$ in length, we selected the final 10 images obtained during the experiment described in Section IV to test the proposed algorithm. Confounding images were simulated by replacing one of the actual transverse US images with a blank image containing "false" needle point candidates placed randomly within the ROI. The image chosen to be replaced was randomized as well. The number of false needle point candidates to be inserted was selected as the average number of needle point candidates found in the remaining "true" images of the needle. Each false needle point candidate had the maximum intensity value in the grayscale range for simplicity. Simulations were performed to test the effects of 0 confounding images up to 9 confounding images. For each case, 100 simulations were performed and the effects on needle shape accuracy and simulation precision were recorded. A graph demonstrating the effects of confounding slices on needle shape accuracy is shown in Fig. 11a. The simulation precision represents the percentage of false needle point candidates included within the consensus set, and is shown in Fig. $11 b$.

Based on the results, the proposed algorithm can function sufficiently up to and including 7 confounding images, making it very robust to problems encountered in clinical US images. After this point, Fig. 11b shows that the precision degrades rapidly, which adversely affects the needle shape accuracy. Note that our proposed algorithm requires a minimum of 2 transverse images to operate properly. However, based on the results of Fig. 11, it appears that the use of 3 or more "relevant" transverse images obtained within $50 \mathrm{~mm}$ of the needle tip allows for more consistent needle shape estimations. Assuming that $33 \%$ of clinical transverse US images will have one of the issues demonstrated in Fig. 10, an average of 5 images would be necessary to obtain accurate results in clinical settings. This is a conservative estimate, since our proposed algorithm is robust against issues such as multiple or distorted needle-like objects that inhibit manual needle segmentation.

\section{Applications to Prostate Brachytherapy}

In this section, we discuss application of the proposed method to PPB.

\section{A. Clinical Setup}

A group of 17 patients undergoing PPB participated in our study ${ }^{1}$. Sample statistics of the patients are shown in Table I. Transverse US images are obtained during the PPB procedures, which are used as inputs to the proposed method. For each patient, 1 to 4 needles are used for the study, yielding 49 needles in total. An experienced brachytherapy surgeon captured a set of 2D transverse US images for each inserted needle using a TRUS probe, Type 8848 (BK Medical, Peabody, MA, USA). The images are captured at $5 \mathrm{~mm}$ intervals using a manually operated Civco Classic Stepper (Civco, Orange City, IA, USA). The images are spaced as such in order to match the template grid spacing, which is $5 \mathrm{~mm}$ both horizontally and vertically. This is a well-established clinical geometry used in prostate brachytherapy equipment and for seed placement planning. The images begin at the needle tip and span the entire prostate, resulting in a set of 10 to 13 images, depending on the length of the patient's prostate. In order to locate the needle tip, the surgeon monitored the US near the prostate base and looked for a "hamburger" signature, a special double reflection artifact that is caused by the bevelled needle tip. A 2D sagittal US image is also obtained for each needle and is used to compare the results from the proposed method for verification. Each sagittal image captured a $50 \mathrm{~mm}$ to $60 \mathrm{~mm}$ needle segment which included the needle tip. The 3D needle shape was calculated using the steps outlined in Section $\mathrm{V}$ and its $2 \mathrm{D}$ projection in the sagittal image plane is compared to the sagittal US image obtained by the surgeon.

\section{B. Projection of 3D Needle Shape onto Sagittal Image Plane}

Before the 3D needle shape estimated using the proposed algorithm can be compared to the sagittal image obtained clinically, we must first project the needle shape estimate onto the sagittal image plane. Because the TRUS probe is cylindrical, all sagittal imaging planes pass directly through the center of the probe. The probe's cross-sectional radius is 9.3 $\mathrm{mm}$, and so the center of the probe can be easily determined in the US image. Next, we estimate the angle of the sagittal image plane for each of the transverse US images, denoted as $\theta_{j}$, where $j$ refers to the index of the transverse image. An

\footnotetext{
${ }^{1}$ Approval for this study granted by the Alberta Cancer Research ethics committee under file number 25837
} 
TABLE I

PATIENT SAMPle Statistics

\begin{tabular}{ccc}
\hline Number of Patients & \multicolumn{2}{c}{17} \\
\hline \multirow{2}{*}{ Age } & Median: & 63 \\
\cline { 2 - 3 } & Range: & $51-69$ \\
\hline \multirow{2}{*}{ Gleason Score } & $6:$ & $12(70.6 \%)$ \\
\cline { 2 - 3 } Pretreatment PSA [ng/mL] & $7:$ & $5(29.4 \%)$ \\
\cline { 2 - 3 } & Median: & 8.3 \\
\hline \multirow{2}{*}{ Cancer Stage } & Range: & $3-12.9$ \\
\cline { 2 - 3 } & T1c: & $7(41.1 \%)$ \\
\cline { 2 - 3 } & T2a: & $8(47.1 \%)$ \\
\cline { 2 - 3 } & T2b: & $1(5.9 \%)$ \\
\hline \multirow{2}{*}{ Risk Category } & T2c: & $1(5.9 \%)$ \\
\cline { 2 - 3 } & Low: & $8(47.1 \%)$ \\
\hline
\end{tabular}

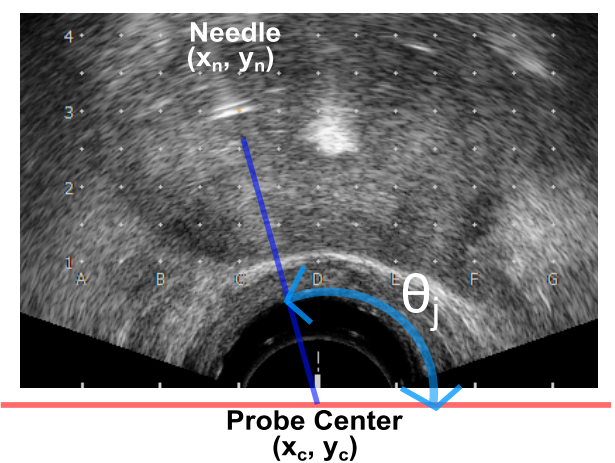

Fig. 12. Demonstration of the sagittal image plane angle estimated from a transverse US image.

example for a single transverse US image is shown in Figure 12. $\theta_{j}$ can be calculated as

$$
\theta_{j}=\left\{\begin{array}{cl}
\arctan \frac{y_{n}-y_{c}}{x_{n}-x_{c}}, & x_{n}>x_{c} \\
\pi+\arctan \frac{y_{n}-y_{c}}{x_{n}-x_{c}}, & x_{n}<x_{c} \\
\frac{\pi}{2}, & x_{n}=x_{c}
\end{array}\right.
$$

where $\left(x_{n}, y_{n}\right)$ represents the needle point estimation obtained using the method described in Section II and $\left(x_{c}, y_{c}\right)$ represents the center of the US probe. The estimated angle of the sagittal image plane will be the average of all $\theta_{j}$ values for the same needle.

The estimated sagittal imaging plane is calculated for each transverse image and the mean angle is obtained. The mean angle is used to derive the transformation matrix $T_{p}$ which is used to convert the 3D needle points $\left(\vec{x}_{3 D}, \vec{y}_{3 D}, \vec{z}_{3 D}\right)$ to their 2D projection $\left(\vec{x}_{\text {proj }}, \vec{y}_{\text {proj }}\right)$ onto the estimated sagittal plane. The transformation is shown below:

$$
\begin{gathered}
{\left[\begin{array}{ccc}
x_{\text {proj,1 }} & \ldots & x_{\text {proj,n }} \\
y_{\text {proj,1 }} & \ldots & y_{\text {proj,n }} \\
z_{\text {proj,1 }} & \ldots & z_{\text {proj,n }} \\
1 & \ldots & 1
\end{array}\right]=T_{\text {proj }}\left[\begin{array}{ccc}
x_{3 D, 1} & \ldots & x_{3 D, n} \\
y_{3 D, 1} & \ldots & y_{3 D, n} \\
y_{3 D, 1} & \ldots & y_{3 D, n} \\
1 & \ldots & 1
\end{array}\right]} \\
T_{\text {proj }}=\left[\begin{array}{cccc}
\cos \theta & \sin \theta & 0 & 0 \\
0 & 0 & 1 & 0 \\
-\sin \theta & \cos \theta & 0 & 0 \\
0 & 0 & 0 & 1
\end{array}\right]
\end{gathered}
$$

TABLE II

Summary Statistics of MANUAL COMPARISON RESUlts

\begin{tabular}{cccc}
\hline & \multicolumn{3}{c}{ Entire Transverse Image Set } \\
\cline { 2 - 4 } & Error: $x$-axis & Error: $y$-axis & Resultant Error \\
\hline Mean [mm] & 0.3 & 0.2 & 0.4 \\
\hline Std. Dev. [mm] & 0.3 & 0.4 & 0.5 \\
\hline & \multicolumn{3}{c}{ 5 Transverse Images } \\
\cline { 2 - 4 } & Error: x-axis & Error: y-axis & Resultant Error \\
\hline Mean [mm] & 0.4 & 0.3 & 0.5 \\
\hline Std. Dev. [mm] & 0.5 & 0.6 & 0.8 \\
\hline
\end{tabular}

Here $n$ is the total number of points defining the $3 \mathrm{D}$ needle shape. We refer to this $2 \mathrm{D}$ projection as the projected needle shape. The needle shape obtained from the sagittal US image will be referred to as the sagittal needle shape.

The needle shape computations described in Sections II and III were applied to the clinical US images using a $2.6 \mathrm{GHz}$ AMD Phenom II X4 910 processor with 4 GB of memory running 32 bit MATLAB. The mean computation times for the image processing (per image) and the needle shape estimation portions of the algorithm were $22 \mathrm{~ms}$ and $351 \mathrm{~ms}$ respectively. The needle shape estimation was calculated using the entire set of transverse images obtained for each needle studied and a minimum of 100 consensus set iterations. The computation times show that the needle shape estimation can be obtained and displayed in real-time for clinical applications and will not impact the overall procedure time.

\section{Needle Shape Comparison Results and Discussion}

Needle point estimation results were compared to manual segmentation using the transverse US image sets obtained from the patient study. These images were selected based on how readily the needle could be manually identified within the image. The needle location was manually identified in each of the transverse US images and the result was compared to two separate applications of the proposed algorithm. In the first application, the entire transverse image set was used as an input to the proposed algorithm. In the second application, the 5 transverse images obtained nearest to the needle tip were used as inputs. The latter application demonstrates how the proposed method functions using a limited set of images, some of which may contain confounding information. Summary statistics are shown in Table II. Using the entire image set, the resultant error between the manual segmentation results and the proposed method averages $0.4 \mathrm{~mm}$, which equates to only 2 pixels in the transverse US image. With the image set of 5 transverse images, the resultant error averages 0.5 $\mathrm{mm}$, which equates to 3 pixels in the transverse US image. In both cases, the needle shape estimation results obtained using the proposed algorithm averaged close to $0.5 \mathrm{~mm}$ of the manual needle segmentation results. Note that manual segmentation involves a degree of human subjectivity and imprecision, which accounts for the vast majority of the smaller errors shown in Table II. Our method provides a consistent, systematic way to locate the needle based on the estimated needle position observed within a series of images. 


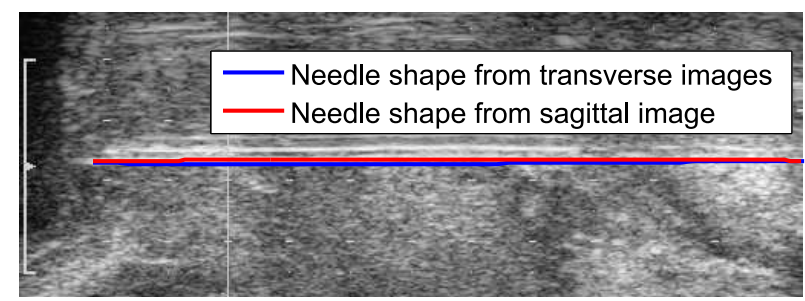

Fig. 13. Comparison of the needle shapes obtained using the transverse and sagittal US images.

TABLE III

Summary Statistics of SAgItTAl IMAge Comparison Results

\begin{tabular}{ccccc}
\hline & \multicolumn{2}{c}{ Entire Transverse Set } & \multicolumn{2}{c}{ 5 Transverse Images } \\
\cline { 2 - 5 } & Max Error & Avg. Error & Max Error & Avg. Error \\
\hline Mean $[\mathrm{mm}]$ & 0.70 & 0.37 & 0.91 & 0.43 \\
\hline Std. Dev. $[\mathrm{mm}]$ & 0.32 & 0.15 & 0.67 & 0.21 \\
\hline
\end{tabular}

The projected needle shape was also compared to the needle segment observed in the sagittal US image as a second way to validate the results. For each sagittal US image, the image processing algorithm described in Section II is applied, and the RANSAC algorithm described in Section III is slightly modified to fit a second-order polynomials to the needle segment. A second-order polynomial was used because the portion of the needle observed in the sagittal US images is approximately half the inserted length. The needle tip was localized in the sagittal images using a similar method to [11], where the needle tip is marked by a drop-off in pixel intensity below the intensity threshold $\alpha$ defined in Section II-C. In addition, the 18 gauge brachytherapy needles used are not as flexible as alternatives used in the literature like nitinol wire. A second-order polynomial is sufficient to estimate this needle segment, as opposed to a third-order polynomial which is reserved for estimation of the entire needle shape.

The sagittal and projected needle shapes are aligned at the point closest to the needle base in the sagittal image. An example of the two needle shapes superimposed over the original image is shown in Fig. 13.

The maximum error between the two needle shapes is measured along with the average error between the estimated needle shape (obtained from the transverse US images) and the reference needle shape (obtained from the sagittal US image). Summary statistics for all 49 needles are presented in Table III.

Based on the results in Table III, we observe that the maximum error between the two needle shapes averages 0.70 $\mathrm{mm}$ when using the entire transverse image set and 0.91 $\mathrm{mm}$ when using only 5 transverse images, both of which are less than the outer diameter of an 18 gauge brachytherapy needle $(1.27 \mathrm{~mm})$. The average errors of $0.37 \mathrm{~mm}$ for the entire transverse image set and $0.43 \mathrm{~mm}$ for the 5 transverse image set corresponds to an errors of 2.1 pixels and 2.4 pixels in the clinical transverse US images respectively, which is very minimal. An average accuracy of $<0.5 \mathrm{~mm}$ is sufficient for clinical applications, where, based on our clinical study, surgeons can tolerate errors of up to $5 \mathrm{~mm}$ with respect to the needle's target position. These results indicate that the proposed method can provide accurate estimates of the needle shape under clinical conditions. This method allows clinicians to use transverse US images to develop an estimate of the 3D needle shape quickly without requiring 3D ultrasound or sagittal images. In addition, the quality of the needle shape information provided by the proposed method is much better than the information surgeons currently use to verify needle placement, which is typically the lateral error observed in a single tranverse US image.

\section{CONCLUSION}

In this paper, a method for 3D needle shape estimation using a discrete series of transverse images is described. Experiments are performed to identify the depth, number, and quality of transverse images required to obtain accurate results. As well, application of the proposed method to patient data is performed and verified by comparing the estimation results to images obtained from the sagittal perspective and to manual segmentation of the transverse US images.

Experimental results showed that to obtain maximum errors of less than $2 \mathrm{~mm}$, the depth of the final image within the transverse image set must be at least $75 \%$ of the maximum needle depth into tissue. Additional tests showed that using 10 transverse images closest to the needle tip, the proposed method is functional as long as the needle can be identified in $30 \%$ of the images obtained. Our method is robust against many of the common US characteristics that confuse both manual and automated segmentation methods.

The proposed method was also applied to in-vivo images obtained from prostate brachytherapy procedures. Two different tests were performed. In the first test, each needle shape was estimated using the entire set of in-vivo transverse US images obtained. In the second test, each needle shape was estimated using the 5 transverse US images obtained nearest to the needle tip. The results were compared to manual segmentation of the same images. The error between the manually segmented results and the results obtained using the proposed method was $0.4 \mathrm{~mm}$ when using the entire transverse US image sets and $0.5 \mathrm{~mm}$ when using the 5 transverse US image sets. The needle shape estimations for each case were also compared to sagittal images obtained during the brachytherapy procedure. Comparisons yielded a maximum error averaging $0.70 \mathrm{~mm}$ when using the entire transverse image sets and $0.91 \mathrm{~mm}$ when using the 5 transverse image sets. A study of computation times showed that the result could be accurately obtained in real-time for operating room conditions.

With respect to the clinical US images, although the comparison could only be performed on the segment of the needle observed in the sagittal US plane, this segment normally spans the entire length of the prostate or slightly greater. Therefore, the proposed method is shown to provide excellent results for the needle shape observed within the prostate, which is normally the segment of greatest interest for determining the dose distribution. The average errors observed in the clinical US images were slightly higher than those obtained in the in-vitro experiments, which could be caused by larger degree 
of noise and hyperechoic objects found when imaging human tissue compared to the plastisol tissue phantom.

\section{REFERENCES}

[1] P. M. Novotny, J. A. Stoll, N. V. Vasilyev, P. J. Del Nido, P. E. Dupont, T. E. Zickler, and R. D. Howe, "GPU based real-time instrument tracking with three-dimensional ultrasound," Medical image analysis, vol. 11, no. 5, pp. 458-464, 2007.

[2] H. Zhou, W. Qiu, M. Ding, and S. Zhang, "Automatic needle segmentation in 3D ultrasound images using 3D improved Hough transform," in Medical Imaging. International Society for Optics and Photonics, 2008, pp. 691821-691821.

[3] W. Qiu, M. Yuchi, M. Ding, D. Tessier, and A. Fenster, "Needle segmentation using 3D Hough transform in 3D TRUS guided prostate transperineal therapy," Medical physics, vol. 40, no. 4, p. 042902, 2013.

[4] M. Ding, Z. Wei, L. Gardi, D. B. Downey, and A. Fenster, "Needle and seed segmentation in intra-operative 3D ultrasound-guided prostate brachytherapy," Ultrasonics, vol. 44, pp. e331-e336, 2006.

[5] W. Qiu, M. Yuchi, and M. Ding, "Phase grouping-based needle segmentation in 3-d trans-rectal ultrasound-guided prostate trans-perineal therapy," Ultrasound in medicine \& biology, vol. 40, no. 4, pp. 804 816, 2014.

[6] H. R. S. Neshat and R. V. Patel, "Real-time parametric curved needle segmentation in 3D ultrasound images," in Biomedical Robotics and Biomechatronics, 2008. BioRob 2008. 2nd IEEE RAS \& EMBS International Conference on. IEEE, 2008, pp. 670-675.

[7] M. Aboofazeli, P. Abolmaesumi, P. Mousavi, and G. Fichtinger, "A new scheme for curved needle segmentation in three-dimensional ultrasound images," in Biomedical Imaging: From Nano to Macro, 2009. ISBI'09. IEEE International Symposium on. IEEE, 2009, pp. 1067-1070.

[8] Z. Wei, L. Gardi, D. B. Downey, and A. Fenster, "Oblique needle segmentation for 3D TRUS-guided robot-aided transperineal prostate brachytherapy," in Biomedical Imaging: Nano to Macro, 2004. IEEE International Symposium on. IEEE, 2004, pp. 960-963.

[9] M. Ding and A. Fenster, "A real-time biopsy needle segmentation technique using Hough transform," Medical physics, vol. 30, no. 8, pp. 2222-2233, 2003.

[10] M. Uhercik, J. Kybic, H. Liebgott, and C. Cachard, "Model fitting using RANSAC for surgical tool localization in 3-d ultrasound images," Biomedical Engineering, IEEE Transactions on, vol. 57, no. 8, pp. 19071916, 2010.

[11] M. Barva, J. Kybic, J.-M. Mari, C. Cachard, and V. Hlavác, "Automatic localization of curvilinear object in 3D ultrasound images," in Medical Imaging. International Society for Optics and Photonics, 2005, pp. 455-462.

[12] Y. Zhao, H. Liebgott, and C. Cachard, "Tracking biopsy needle using Kalman filter and RANSAC algorithm with 3D ultrasound," Acoustics 2012 Nantes, 2012.

[13] K. J. Chin, A. Perlas, V. W. Chan, and R. Brull, "Needle visualization in ultrasound-guided regional anesthesia: challenges and solutions," Regional anesthesia and pain medicine, vol. 33, no. 6, pp. 532-544, 2008.

[14] G. Chapman, D. Johnson, and A. Bodenham, "Visualisation of needle position using ultrasonography," Anaesthesia, vol. 61, no. 2, pp. 148$158,2006$.

[15] M. Kaya and O. Bebek, "Needle localization using gabor filtering in 2D ultrasound images," in Robotics and Automation (ICRA), 2014 IEEE International Conference on. IEEE, 2014, pp. 4881-4886.

[16] _ _ "Gabor filter based localization of needles in ultrasound guided robotic interventions," in Imaging Systems and Techniques (IST), 2014 IEEE International Conference on, Oct 2014, pp. 112-117.

[17] S. H. Okazawa, R. Ebrahimi, J. Chuang, R. N. Rohling, and S. E. Salcudean, "Methods for segmenting curved needles in ultrasound images," Medical image analysis, vol. 10, no. 3, pp. 330-342, 2006.

[18] A. Ayvaci, P. Yan, S. Xu, S. Soatto, and J. Kruecker, "Biopsy needle detection in transrectal ultrasound," Computerized Medical Imaging and Graphics, vol. 35, no. 7, pp. 653-659, 2011.

[19] K. Mathiassen, D. Dall'Alba, R. Muradore, P. Fiorini, and O. J. Elle, "Real-time biopsy needle tip estimation in 2D ultrasound images," in Robotics and Automation (ICRA), 2013 IEEE International Conference on. IEEE, 2013, pp. 4363-4369.

[20] Z. Neubach and M. Shoham, "Ultrasound-guided robot for flexible needle steering," Biomedical Engineering, IEEE Transactions on, vol. 57, no. 4, pp. 799-805, 2010.
[21] J. Carriere, C. Rossa, R. Sloboda, N. Usmani, and M. Tavakoli, "Needle shape estimation in soft tissue based on partial ultrasound image observation," in Robotics \& Automation (ICRA), 2015 IEEE International Conf. on, May 2015.

[22] B. J. Davis, E. M. Horwitz, W. R. Lee, J. M. Crook, R. G. Stock, G. S Merrick, W. M. Butler, P. D. Grimm, N. N. Stone, L. Potters et al., "American brachytherapy society consensus guidelines for transrectal ultrasound-guided permanent prostate brachytherapy," Brachytherapy, vol. 11, no. 1, pp. 6-19, 2012.

[23] J. D. Greer, T. K. Adebar, G. L. Hwang, and A. M. Okamura, "Realtime 3D curved needle segmentation using combined b-mode and power doppler ultrasound," in Medical Image Computing and ComputerAssisted Intervention-MICCAI 2014. Springer, 2014, pp. 381-388.

[24] T. Adebar, A. Fletcher, and A. Okamura, "3D ultrasound-guided robotic needle steering in biological tissue," IEEE Transactions on Biomedical Engineering, vol. 61, no. 12, pp. 2899-2910, 2014.

[25] G. J. Vrooijink, M. Abayazid, and S. Misra, "Real-time threedimensional flexible needle tracking using two-dimensional ultrasound," in Robotics and Automation (ICRA), 2013 IEEE International Conference on. IEEE, 2013, pp. 1688-1693.

[26] G. J. Vrooijink, M. Abayazid, S. Patil, R. Alterovitz, and S. Misra, "Needle path planning and steering in a three-dimensional non-static environment using two-dimensional ultrasound images," The International Journal of Robotics Research, p. 0278364914526627, 2014.

[27] P. Yan, J. C. Cheeseborough, and K. C. Chao, "Automatic shape-based level set segmentation for needle tracking in 3-d TRUS-guided prostate brachytherapy," Ultrasound in medicine \& biology, vol. 38, no. 9, pp. 1626-1636, 2012.

[28] M. Waine, C. Rossa, R. Sloboda, N. Usmani, and M. Tavakoli, "3D needle shape visualization from 2D ultrasound images using RANSAC," in Robotics \& Automation (ICRA), 2015 IEEE International Conf. on, May 2015.

[29] R. Roesthuis, Y. R. van Veen, A. Jahya, and S. Misra, "Mechanics of needle-tissue interaction," in Intelligent Robots and Systems (IROS), 2011 IEEE/RSJ International Conference on, Sept 2011, pp. 2557-2563.

[30] X. Wen, S. Salcudean, and P. Lawrence, "Detection of brachytherapy seeds using 3-d transrectal ultrasound," Biomedical Engineering, IEEE Transactions on, vol. 57, no. 10, pp. 2467-2477, Oct 2010.

[31] R. C. Gonzalez, R. E. Woods, and S. L. Eddins, Digital Image Processing Using MATLAB. Upper Saddle River, NJ, USA: Prentice Hall Press, 2007.

[32] P. Perona and J. Malik, "Scale-space and edge detection using anisotropic diffusion," Pattern Analysis and Machine Intelligence, IEEE Transactions on, vol. 12, no. 7, pp. 629-639, 1990.

[33] Y. Yu and S. T. Acton, "Speckle reducing anisotropic diffusion," Image Processing, IEEE Transactions on, vol. 11, no. 11, pp. 1260-1270, 2002.

[34] S. Aja-Fernández and C. Alberola-López, "On the estimation of the coefficient of variation for anisotropic diffusion speckle filtering," Image Processing, IEEE Transactions on, vol. 15, no. 9, pp. 2694-2701, 2006.

[35] M. Abayazid, R. J. Roesthuis, R. Reilink, and S. Misra, "Integrating deflection models and image feedback for real-time flexible needle steering," Robotics, IEEE Transactions on, vol. 29, no. 2, pp. 542-553, 2013.

[36] R. Alterovitz, K. Goldberg, and A. Okamura, "Planning for steerable bevel-tip needle insertion through $2 \mathrm{D}$ soft tissue with obstacles," in Robotics and Automation, 2005. ICRA 2005. Proceedings of the 2005 IEEE International Conference on. IEEE, 2005, pp. 1640-1645.

[37] K. B. Reed, A. Majewicz, V. Kallem, R. Alterovitz, K. Goldberg, N. J. Cowan, and A. M. Okamura, "Robot-assisted needle steering," Robotics \& Automation Magazine, IEEE, vol. 18, no. 4, pp. 35-46, 2011.

[38] M. A. Fischler and R. C. Bolles, "Random sample consensus: a paradigm for model fitting with applications to image analysis and automated cartography," Communications of the ACM, vol. 24, no. 6, pp. 381-395, 1981.

[39] M. Zuliani, "RANSAC toolbox for MATLAB," [web page] http://www.mathworks.com/matlabcentral/fileexchange/18555, Nov. 2008, [Accessed on: August 1, 2014].

[40] P. H. Torr and A. Zisserman, "MLESAC: A new robust estimator with application to estimating image geometry," Computer Vision and Image Understanding, vol. 78, no. 1, pp. 138-156, 2000. 